\title{
Local Adaptive Contrast Enhancement for Color Images
}

\author{
Judith Dijk, Richard J.M. den Hollander, John G.M. Schavemaker and Klamer Schutte \\ TNO Defence, Security and Safety \\ P.O. Box 96864, 2509 JG The Hague, The Netherlands \\ Email: Judith.Dijk@tno.nl
}

\begin{abstract}
A camera or display usually has a smaller dynamic range than the human eye. For this reason, objects that can be detected by the naked eye may not be visible in recorded images. Lighting is here an important factor; improper local lighting impairs visibility of details or even entire objects. When a human is observing a scene with different kinds of lighting, such as shadows, he will need to see details in both the dark and light parts of the scene. For grey value images such as IR imagery, algorithms have been developed in which the local contrast of the image is enhanced using local adaptive techniques. In this paper, we present how such algorithms can be adapted so that details in color images are enhanced while color information is retained. We propose to apply the contrast enhancement on color images by applying a grey value contrast enhancement algorithm to the luminance channel of the color signal. The color coordinates of the signal will remain the same. Care is taken that the saturation change is not too high. Gamut mapping is performed so that the output can be displayed on a monitor. The proposed technique can for instance be used by operators monitoring movements of people in order to detect suspicious behavior. To do this effectively, specific individuals should both be easy to recognize and track. This requires optimal local contrast, and is sometimes much helped by color when tracking a person with colored clothes. In such applications, enhanced local contrast in color images leads to more effective monitoring.
\end{abstract}

Keywords: Local contrast, image enhancement, adaptive, color

\section{INTRODUCTION}

A camera or display usually has a smaller dynamic range than the human eye. This may cause that objects that can be detected in the original scene by the naked eye are not visible in images displaying the same scene. However, in many cases these objects or more general information that is not visible can still be recovered from the image data.

Lighting plays an important role in the visibility of objects in images. When a human is observing a scene with different kinds of lighting, such as shadows, he will need to see details in both the dark and light parts of the scene. Human eyes are capable to do so, but when this image is recorded and displayed some of the details may be gone. In general, improper lighting may cause details or even entire objects in image to be invisible, even if the human could see it in the original scene.

Enhancing the contrast in color images can for instance be used by humans monitoring movements of people on a display in order to detect suspicious behavior. To do this effectively, specific individuals should be as easy to recognize and track as possible. This requires optimal local contrast, and is sometimes much helped by color for instance, when tracking a person who is wearing colored clothes. In such applications, enhanced local contrast in color images leads to more effective monitoring.

Examples of images with different lighting conditions are shown in figure 1. The first image, figure 1(a) is a frame from a surveillance camera. In this image two men are seen, one on the outside and one inside the building. For surveillance, the actions of both men should be visible. The second image, in figure 1(b) is an image from a lock. The operator should see all boats and ships and also if the lock is properly filled. However, on this image there is a large shadow.

For grey value images such as IR imagery, algorithms have been developed in which the local contrast of the image is enhanced by using local adaptive techniques. In section 2 an overview of these algorithms is given.

Visual Information Processing XVI, edited by Zia-ur Rahman, Stephen E. Reichenbach, Mark Allen Neifeld, Proc. of SPIE Vol. 6575, 65750A, (2007) · 0277-786X/07/\$18 · doi: 10.1117/12.716886 
For color images there are also situations where we want to enhance the details in the image. But in most situations we would also like to keep the color, as also the color gives important information about objects in the scene.

In this paper we present how grey-value algorithms can be adapted so that the details in color images are enhanced by applying the contrast enhancement to the luminance channel of the color signal. The color coordinates of the signal remain the same. The proposed technique can be implemented on a real-time system.

The contrast in displayed images can also be increased by using special displays with a high dynamic range. However, the extra dynamic range may be insufficient to enhance all local contrasts. These special displays are also rather expensive, where our solution can be used on standard monitors.

This paper is organized as follows. In section 2 other methods for local contrast enhancement are discussed. In section 3 the proposed algorithm is described, and in section 4 results with these algorithms are shown. In the last section the conclusions are given and issues for new research are discussed.

\section{LITERATURE}

\subsection{Local contrast enhancement in grey value images}

To enhance the contrast in an image several methods are described in literature. ${ }^{1-7}$ The most simple way to enhance the contrast is by adjusting the image to the available range, so-called global contrast stretching. This will help in cases were only part of the available range is used, but will fail in situations were the range used in one part of the image is very different than in another. To enhance the contrast in a part of the available contrast range a global gamma correction can be done. In this case details in part of the luminance range of the image will be enhanced, at the cost of decreasing the luminance in other parts of the image. Another global method is histogram equalization, were the luminance values are changed so that the histogram of the image are as flat as possible. The main disadvantage of this method is that the output images are not so natural anymore.

To enhance local contrasts grey-value local adaptive contrast enhancement can be used. The idea of local contrast enhancement is that the processing depends features in a local region. Narenda and Fitch ${ }^{2}$ propose a method in which the local statistics are described by its local mean and variance. The difference between the local mean and original pixel value is added adaptively to the result,

$$
o=i+f(m-i)
$$

where $o$ is the output image, $i$ the original image, $m$ the local mean and $f$ a function based on the local statistics.

$$
f(s)=\alpha \frac{M}{s}-1
$$

where $\mathrm{M}$ is the global mean in the image and $s$ is the local standard deviation.

Using a local region means that the contrast is enhanced in a specific scale in the image, for instance only small details. However, it is hard to decide which details are important and which are not. Most authors suggest therefore that one the contrast should be adjusted at several scales in the image. To do so, either different scales can be determined or a multi-scale pyramid can be constructed.

It is also possible to perform gamma correction on a local scale, according to

$$
o(x, y)=255\left(\frac{i(x, y)}{255}\right)^{\gamma(x, y)}
$$

where

$$
\gamma(x, y)=\frac{i_{g}(x, y)-128}{128}
$$


The value of $\gamma$ depends on $i_{g}(x, y)$, which is the Gaussian filtered input image with a Gaussian of appropriate size. When $\gamma$ is chosen $>1$ it provides luminance stretching on the lower and upper parts of the luminance range.

In our evaluation we use a grey-value algorithm ${ }^{7}$ which is based on the local mean and variance in different scales. Here the output image $o$ is derived by

$$
o=i+\sum_{j=1}^{k} c\left(m_{j}, s_{j}\right)\left(i-m_{j}\right)
$$

where $m_{j}$ and $s_{j}$ are the local mean and standard deviation at scale $j . k$ is the total number of scales. The contrast enhancement function at each scale is given by

$$
c\left(m_{j}, s_{j}\right)=\alpha \frac{m_{j}}{s_{j}}-1 .
$$

where $\alpha$ is the weighing factor with the original image. $c\left(m_{j}, s_{j}\right)$ is clipped to $A_{\max }$ on top and zero on the bottom of the range.

This algorithm can be used to obtain images showing all interesting features in an image. An advantage of this algorithm is that it can be implemented on hardware, as well in a real-time software application.

\subsection{Local contrast enhancement in color images}

In literature also methods for color contrast enhancement are described. Toet e.g. describes a method for color image enhancement. ${ }^{8}$ This method increases the luminance contrast of a color image by using both luminance and saturation contrast in the image. Toet constructs two pyramids, one for the luminance component and one for the saturation component of the original color image. Then new luminance primitives in the pyramid are determined, based on the application. For instance, the most significant edges can be taken or saturation edges can be added in the luminance image.

Mukhopadhyay and Chanda ${ }^{9}$ also propose a contrast enhancement method, which preserves the colors by modifying the luminance component only. Multi-scale top-hat transforms on the luminance image are added to the original luminance image to enhance the contrast. This makes that both bright and dark objects of different sizes are being amplified, where the smaller objects are amplified more than the larger objects.

In Starck et al. ${ }^{10}$ the curvelet transform is applied to the individual components of the CIELUV space. The curvelet coefficients of each component are multiplied by a function of $\sqrt{c_{L}^{2}+c_{u}^{2}+c_{v}^{2}}$, where $c_{L}, c_{u}$ and $c_{v}$ are the original curvelet coefficients. The contrast enhanced image is obtained by the inverse curvelet transform applied to the modified coefficients.

\section{ALGORITHM}

In the proposed algorithm we perform the local contrast enhancement on color images by performing the contrast enhancement on the luminance channel, after which we recombine it with its colors. This can be done with every grey-value local contrast enhancement.

The idea of the algorithm is to change the image so that the luminance or grey-value contrast is enhanced, while keeping the saturation and hue more or less the same. Therefore we use the perceptual colors of the CIEXYZ color space. The reason to use the CIEXYZ color space instead of the CIELAB color space, in which the colors are spaced perceptually, is that algorithms based on the CIELAB color space are harder to implement in a real-time system. However, it can be shown that using the current algorithm, the hue in the CIELAB color space does not change. ${ }^{11}$ In the CIEXYZ color space a pixel is represented by its luminance value $Y$ and two chromaticity values $x=X /(X+Y+Z)$ and $y=Y /(X+Y+Z)$. The last two values denote the color of the pixel, irrespective of its lightness. We perform the contrast enhancement on the luminance channel $Y$. The chromaticity values $x$ and $y$ remain the same. To obtain the XYZ values of a pixel when the $x$ and $y$ stay the same, one needs to scale the XYZ values by $d Y=Y_{\text {new }} / Y_{\text {old }}$ 
When converting this to RGB, it can be seen that also the RGB values are scaled with the luminance change. To obtain RGB values from XYZ, the following formula is used

$$
R G B_{\text {old }}=A * X Y Z_{\text {old }}
$$

where $\mathrm{A}$ is a $3 \mathrm{x} 3$ matrix.

$$
R G B_{\text {new }}=A * X Y Z_{\text {new }}=A * d Y X Y Z_{\text {old }}=d Y R G B_{\text {old }}
$$

A problem encountered is that the saturation of some colors can be increased so much, that the color seems to be fluorescent. This was mainly seen in red colors. This problem may be caused by the the choice for the CIEXYZ color space instead of a perceptually changed color space, such as CIELAB, where can be shown that a luminance increase will cause a chroma increase. ${ }^{11}$

To solve the problem, the saturation of the image should not increase too much. This is done in the RGB color space by limiting the luminance scaling by the maximum saturation scaling. If we define the saturation as

$$
\text { sat }=\max (|R-G|,|R-B|,|G-B|)
$$

then the luminance gain $d Y$ is scaled if the change is saturation is larger than a maximum value maxSatChange.

$$
d Y^{\prime}= \begin{cases}d Y & \text { for } \operatorname{sat}(d Y-1)<\max \text { SatChange } \\ \frac{\text { maxSatChange+sat}}{\text { sat }} & \text { for sat }(d Y-1) \geq \max \text { SatChange }\end{cases}
$$

A typical value for the maximum saturation change is 32 .

Before displaying gamut mapping needs to be applied, because due to the scaling the RGB values can be larger than 255. Note that the values can never be smaller than 0 if the input RGB values are already larger than 0 . The gamut mapping is done by mixing the colors with black when necessary, or in formula

$$
d Y^{\prime \prime}=\frac{255 d Y^{\prime}}{\max (R, G, B, 255)}
$$

\section{RESULTS}

In this section we show the results of the proposed algorithm for the two different images shown in figure 1.

By changing the parameters $\alpha$ and $A_{\max }$ different amounts of contrast enhancement can be obtained. In figure 2 and 3 results are shown for both images for low, medium and high contrast enhancement. In both images it can be seen that the contrast is enhanced, in the surveillance image the man standing inside the building is seen much better, whereas in the lock image a boat in the shadows suddenly appears. Increasing the contrast enhancement from low to medium or high will increase the contrast that is seen, but also the noise.

The resulting images are more or less natural. Two artefacts decrease the naturalness of the images. The first is that the grey-value algorithm tends to have some overshoot near large edges. This can for instance be seen near the lamps in the lock image, where blocks appear. The second artefact is that the color of shadows is not exactly grey, so that the restored colors in the shadow area are not so natural. This can for instance be seen in the large shadow inside the lock.

In figure 5 and 4 the difference between the color images and the grey values images are shown. It can be seen that the color images have more relevant information than the grey-value images, such as the color of the skin and the color of the water.

In figure 6 the need for saturation clipping is shown. In the left images no saturation clipping is applied. It can be seen that the car and the live saving belt are much to red. In the right images saturation clipping is applied. Here the car and belt are not fluorescent anymore.

In figure 7 our method is compared to some standard methods. These grey value methods are applied on the luminance of the color image in the same way as our algorithm. The results are shown for a small part of 
the input image so that the details in the boat are better seen. In figure 7(a) the part of the original image is shown. In figure $7(\mathrm{~b})$ the result is shown for histogram equalization. It can be seen that the visibility of the boat is not really improved, as the shadows are still rather dark. When applying global gamma correction (figure $7(\mathrm{c})$ ) the shadow is lighter, but the contrast in the image is also lighter. The local gamma correction gives a rather good result, where the details on the land and in the water are still visible. The output of our algorithm using medium contrast enhancement is shown in $7(\mathrm{e})$. It can be seen that all details are still visible, both on the land as in the water. The details seem to be somewhat more coarse than with the local gamma method. Some details, such as the white objects on the boat, are better seen than in the other methods.

\section{CONCLUSIONS AND DISCUSSION}

In this paper we present a new color local adaptive contrast enhancement algorithm. In this algorithm a grey-value local adaptive contrast enhancement is applied on the luminance component, while keeping the chromaticity values $x$ and $y$ constant. For luminance increases the saturation of some colors increases too much, making the colors somewhat fluorescent. Therefore, the luminance change is limited by a maximum saturation change. As last step gamut mapping is performed so that the output can be displayed on a monitor. This gamut mapping is done by mixing out-of-gamut colors with black.

Results show that the interesting features in the images are enhanced and that the resulting color image is more or less natural. The algorithm is applied on two color images from monitoring applications: a frame of a surveillance camera and a camera in a lock. It is shown that in these applications, enhanced local contrast in color images leads to more effective monitoring. The proposed algorithm can be implemented on a real-time system, which is necessary for such applications.

For color images the naturalness of the output is important. In next studies we will address two artefacts in the current algorithm which decrease the naturalness: reduction of the artefacts around large edges and compensation for the color of the shadows. Another topic we will study is the effect of using the CIEXYZ color space instead of the CIELAB or CIELUV color space, in which distances are more perceptually spaced. We expect that the saturation increase will not be seen in these color spaces. However, as the conversion to these color spaces is computationally more expensive, it is harder to implement such a solution on a real-time system.

\section{REFERENCES}

1. Young, I.T., J.J. Gerbrands, and L.J. van Vliet (1998) Image Processing Fundamentals In: Madisetti, V.K. and B.D. Williams, editors, The Digital Signal Processing Handbook, chapter 51, pages 1-81. IEEE Press and CRC Press.

2. Narenda, P.M. and R.C. Finch (1981) Real-time adaptive contrast enhancement IEEE transactions on pattern analysis and machine intelligence vol. 3, no. 6, pp. 655-661

3. De Vries, F. (1990) Automatic, adaptive, brightness independent contrast enhancement, Signal Processing vol. 12, pp. 169-182

4. Paranjape, R.B., W.M. Morrow and R.M. Rangayyan (1992) Adaptive-neighborhood histogram equalization for image enhancement Computer Vision, Graphics and Image Processing: Graphical models and image processing vol. 52, no. 3, pp. 259-267

5. Mukherjee, D. and B. Chatterji (1990) Adaptive Neighborhood extended contrast enhancement and its modifications Pattern Recognition Letters, vol. 11, pp. 735-742

6. Moroney, M. (2000) Local Color Correction using Non-Linear Masking IS\&T/SID Eight Color Imaging Conference pp 108-111.

7. Schutte, K. (1997) Multi-scale adaptive Gain control of IR images SPIE vol. 3061, pp. 906-914.

8. Toet, A. (1992). Multiscale color image enhancement. Pattern Recognition Letters vol. 13, pp. 167-174

9. Mukhopadhyay, S. and Chanda, B. (2002). Hue preserving color image enhancement using multi-scale morphology. Indian Conference on Computer Vision, Graphics and Image Processing 
10. Starck, J.-L. and Murtagh, F. and Candes, E.J. and Donoho, D.L. (2003) Gray and color image enhancement using the curvelet transform. IEEE transactions on image processing vol. 12, no. 6, pp. 706-717.

11. Dijk, J. (2004) In search of an Objective Measure for the Perceptual Quality of Printed Images PhD thesis, Delft University of Technology 


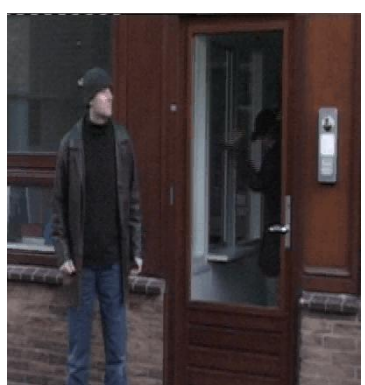

(a) surveillance

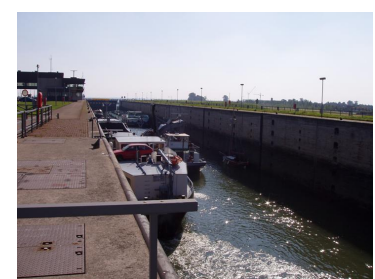

(b) lock

Figure 1. The test images.

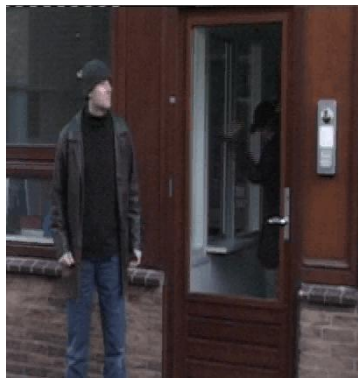

(a) normal

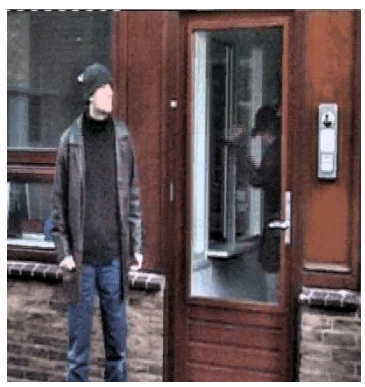

(b) low Contrast Enhancement

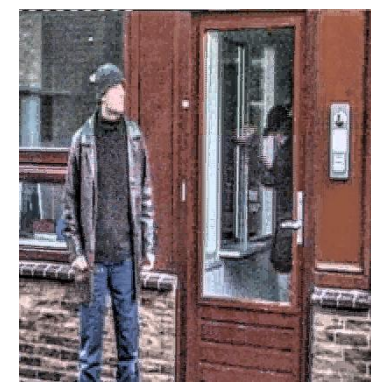

(c) medium Contrast Enhancement

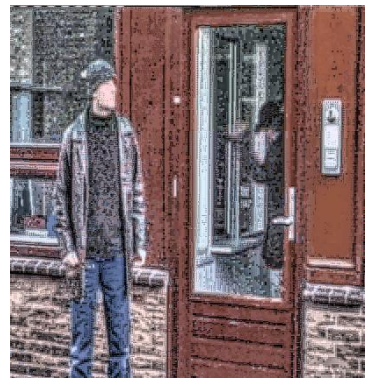

(d) high Contrast Enhancement

Figure 2. Results for the surveillance image.

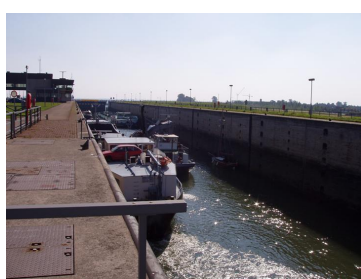

(a) normal

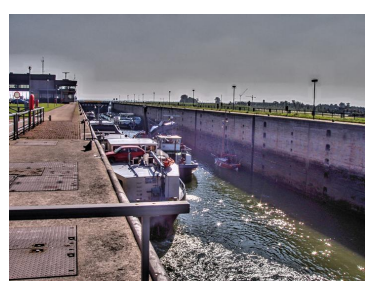

(b) low Contrast Enhancement

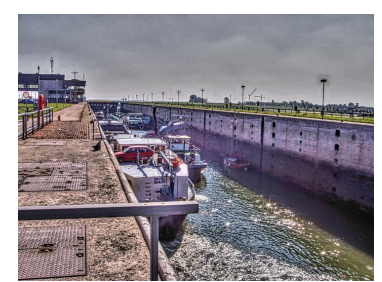

(c) medium Contrast Enhancement

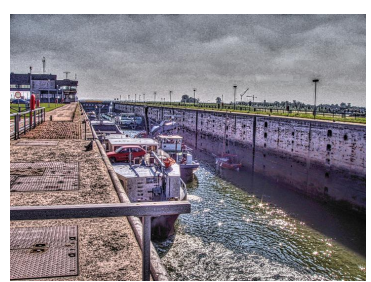

(d) high Contrast Enhancement

Figure 3. Results for the lock image.

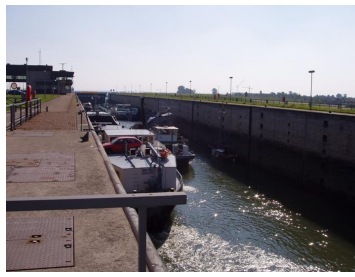

(a) color normal

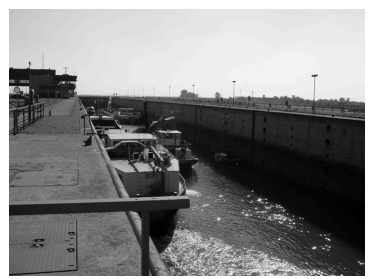

(b) grey normal

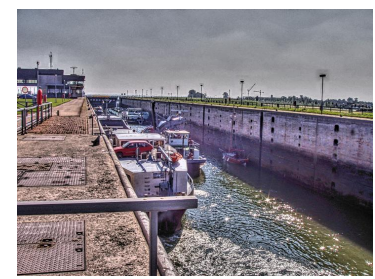

(c) color medium Contrast Enhancement

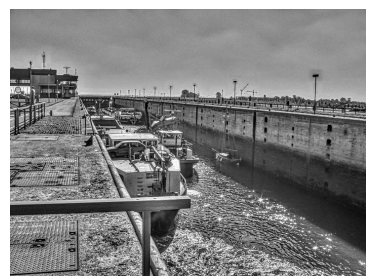

(d) grey medium Contrast Enhancement

Figure 4. Difference between color and grey value processing for the lock image. 


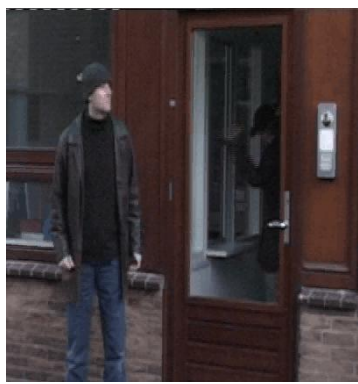

(a) color normal

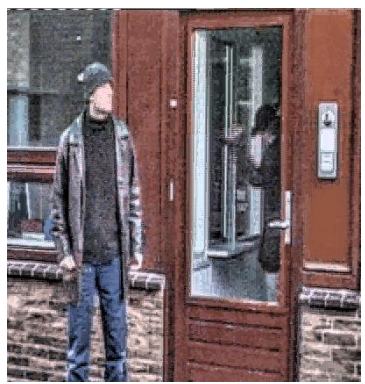

(b) grey normal

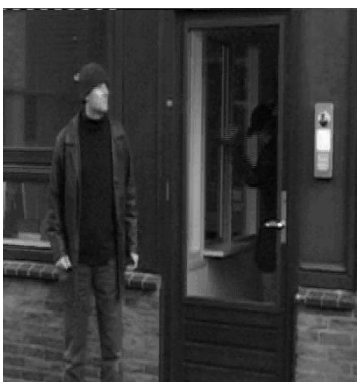

(c) color medium Contrast Enhancement

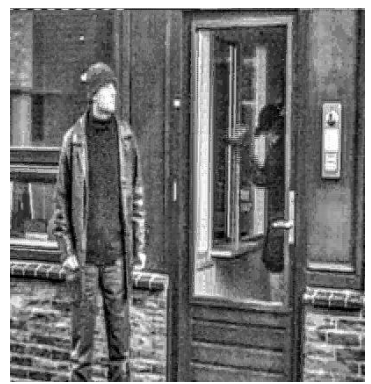

(d) grey medium Contrast Enhancement

Figure 5. Difference between color and grey value processing for the surveillance image.

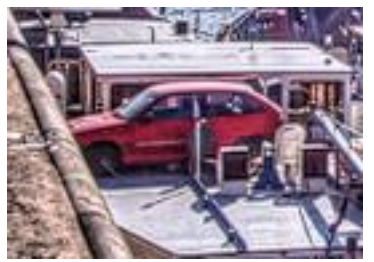

(a) without saturation clipping

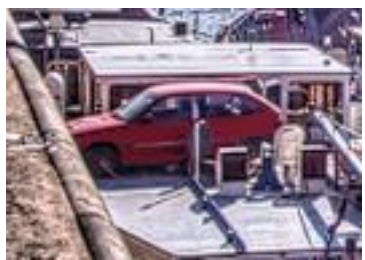

(b) with saturation clipping

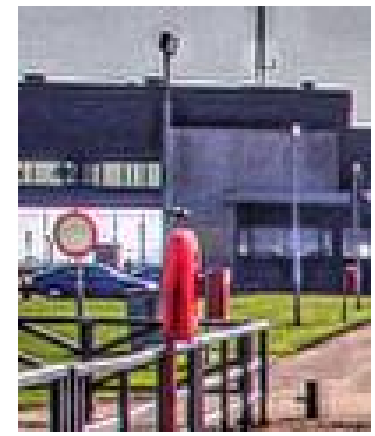

(c) without saturation clipping

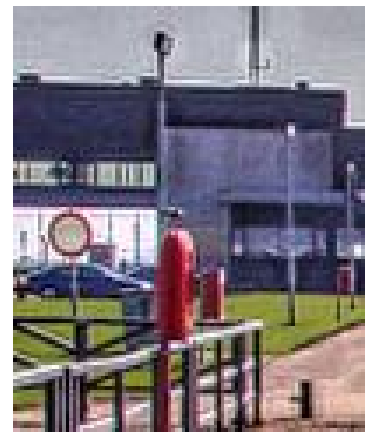

(d) with saturation clipping

Figure 6. Results with and without saturation clipping.

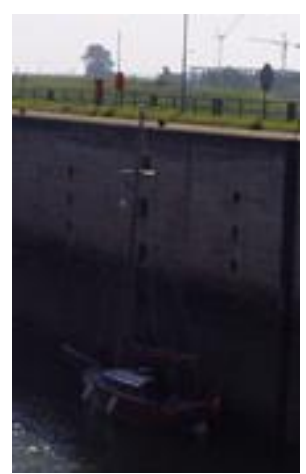

(a) original image

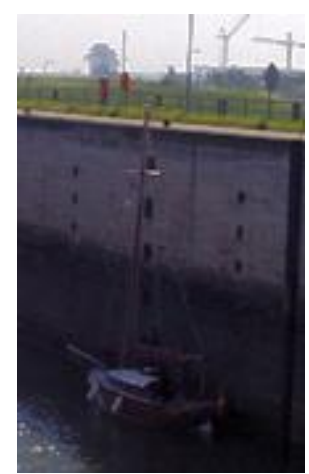

(b) histogram equalization

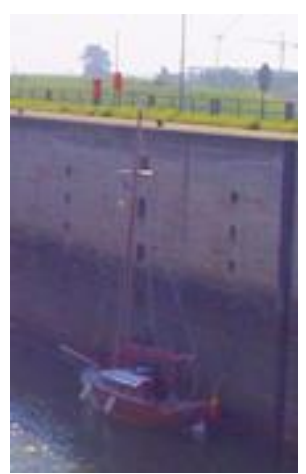

(c) global gamma $(\gamma=0.5)$

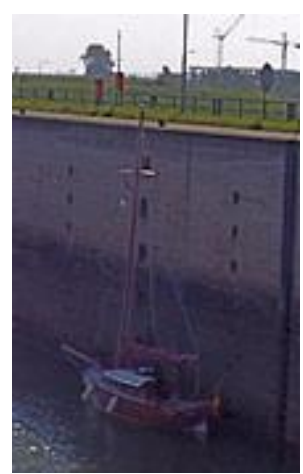

(d) local gamma $(\gamma$ $=3$, scale $=1$ )

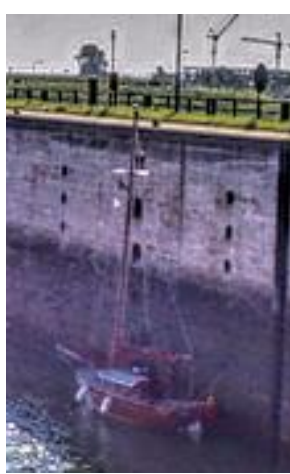

(e) method

Figure 7. Comparison of the results for different contrast enhancement methods. 